\title{
Thrombocytopenia in Pregnancy Nearing Term: A Clinical Analysis
}

\author{
Minal Harde ${ }^{1}$, Rakesh Bhadade ${ }^{2}$, Rosemarie deSouza ${ }^{3}$, Mrida Jhingan ${ }^{4}$
}

\begin{abstract}
Background and aim: Thrombocytopenia in pregnancy varies from benign to severe with fetomaternal complications. We aimed to evaluate thrombocytopenia in pregnant Indian females in third trimester mainly during labor and delivery.

Materials and methods: It was a prospective observational study done in a tertiary care teaching public hospital over 1 year. Consecutive 150 pregnant patients admitted to labor ward with thrombocytopenia were analyzed for etiology of thrombocytopenia, severity, mode of delivery, type of anesthesia, and fetomaternal complications. SPSS version 17 was used for the analysis.

Results: Most common cause of thrombocytopenia was preeclampsia $50(33.3 \%)$ and preeclampsia with hemolysis, elevated liver enzyme, and low platelet count syndrome (HELLP syndrome) 31 (20.7\%) together followed by gestational 42 (28\%). Infectious causes such as malaria, dengue, and leptospirosis were found in 19 patients (12.7\%). Moderate to severe thrombocytopenia was seen in preeclampsia, preeclampsia with HELLP syndrome, and infectious etiology. Eleven patients (7.3\%) developed antepartum hemorrhage (APH), 24 (16\%) postpartum hemorrhage (PPH), 12 (8\%) required ICU admission, and $3(2 \%)$ mortalities were noted. Fifteen neonates (10\%) needed ICU admission. Complications were observed in preeclampsia with HELLP syndrome (82\%) and infectious causes (18\%) and none in gestational. Sixty-eight patients underwent lower segment cesarean section (LSCS), among them 41 (27.3\%) were given spinal anesthesia (SA) and none of them developed any neurological complications. Conclusion: Study widened the spectrum of causes for thrombocytopenia in pregnant patients. Preeclampsia with or without HELLP syndrome and vector-borne infections such as malaria, dengue, and leptospirosis were found to be very important causes of moderate to severe thrombocytopenia and were associated with complications. Spinal anesthesia is safe in parturients with mild thrombocytopenia. Awareness and vigilance about thrombocytopenia is vital to reduce maternal morbidity and mortality.

Keywords: Hemolysis, elevated liver enzyme, and low platelet count syndrome, Infections, Preeclampsia, Spinal anesthesia, Thrombocytopenia in pregnancy.

Indian Journal of Critical Care Medicine (2019): 10.5005/jp-journals-10071-23277
\end{abstract}

\section{INTRODUCTION}

Thrombocytopenia is one of the common findings in pregnancy. It may be benign as gestational thrombocytopenia or severe associated with a risk of fetomaternal morbidity and mortality. The frequent hematological findings in pregnancy are anemia and thrombocytopenia. Thrombocytopenia affects $6.6-11.6 \%$ of all pregnant women. ${ }^{1}$ The normal range of platelets in non-pregnant women is $1,50,000-4,00,000 / \mu \mathrm{L}$. Thrombocytopenia is defined as a drop in platelet count below $1,50,000 / \mu \mathrm{L}$. It can be graded according to severity into mild $(1,00,000-1,50,000)$, moderate $(50,000-1,00,000)$, and severe $(<50,000)$ thrombocytopenia. ${ }^{2}$

The platelet count may decrease by approximately $6-7 \%$ and it generally occurs during third trimester because of hemodilution, increased platelet consumption, and increased platelet aggregation due to increased levels of thromboxane A2. ${ }^{3}$ However, absolute platelet count remains within normal reference range in most patients. Thrombocytopenia during pregnancy may get worse as the pregnancy progresses. ${ }^{4}$ Also thrombocytopenia in nearing-term patients may affect delivery and anesthesia choice.

Causes of thrombocytopenia may be unique to pregnancy or can be medical conditions associated with pregnancy. They range from conditions like gestational thrombocytopenia, immune thrombocytopenia (ITP), preeclampsia, HELLP syndrome, or thrombotic thrombocytopenic purpura (TTP) to various infectious causes. ${ }^{5-8}$ Distinguishing the cause is very crucial since the management differs broadly. Hence, awareness about different causes, early diagnosis, and institution of specific treatment may improve both maternal and neonatal outcomes. The literature
${ }^{1,4}$ Department of Anaesthesiology, Topiwala National Medical College and BYL Nair Ch. Hospital, Mumbai, Maharashtra, India

${ }^{2,3}$ Department of Medicine, Topiwala National Medical College and BYL Nair Ch. Hospital, Mumbai, Maharashtra, India

Corresponding Author: Minal Harde, Department of Anaesthesiology, Topiwala National Medical College and BYL Nair Ch. Hospital, Mumbai, Maharashtra, India, Phone: +91 2223027137, e-mail: minalharde@ yahoo.co.in

How to cite this article: Harde $M$, Bhadade R, deSouza R, Jhingan M. Thrombocytopenia in Pregnancy Nearing Term: A Clinical Analysis. IJCCM 2019;23(11):503-508.

Source of support: Nil

Conflict of interest: None

available in this regard is focused on a particular etiology and there is paucity of data from patients nearing term in Indian population.

Hence, we aimed to study thrombocytopenia in pregnant Indian females in third trimester mainly during labor and delivery with respect to etiology of thrombocytopenia, severity of thrombocytopenia, mode of delivery, type of anesthesia, any complications encountered, requirement of transfusion, and maternal and fetal outcomes.

\section{Materials and Methods}

It was a prospective observational cohort study done in a tertiary care teaching public hospital over a period of 1 year from January 
2013 to February 2014. Consecutive pregnant patients admitted to labor ward were screened for thrombocytopenia and patients with platelet count less than 1.5 lacks were included in the study. Study was conducted after obtaining approval from the Institutional Ethical Committee and written informed valid consent from the patients.

A detailed history, physical examination, and necessary investigations were carried out in all patients as a part of standard protocol and management. We noted following data from the patients chart: maternal age, parity, gestational age at the time of diagnosis and at the time of delivery, diagnosis and cause of thrombocytopenia, mode of delivery like normal vaginal delivery or LSCS, type of anesthesia given for LSCS whether SA or general anesthesia (GA), requirement of platelet transfusion, any complications like $\mathrm{APH}, \mathrm{PPH}$, requirement of ICU admission, and mortality were noted till discharge. We also noted outcome of fetus and requirement of NICU admission.

\section{Statistical Analysis}

Qualitative data were represented in the form of frequency and percentage. Quantitative data were represented using mean $\pm S D$, median, and interquartile range (IQR). Association between qualitative variables was assessed by Chi-square test with continuity. Standard statistical methods such as ANOVA and student's " $t$ " test were used to find the association between different causes and severity of thrombocytopenia. SPSS version 17 was used for the analysis.

\section{Results}

During the study period of 1 year, total patients admitted in labor ward were 1,875, and 150 patients were diagnosed with thrombocytopenia (incidence 8\%). Mean age of patients was 25.27 years. Average gestational age at diagnosis was 35.66 weeks and at delivery was 37.96 weeks. Mean platelet count at diagnosis was 97,712 and at delivery was 95,309 (Table 1).

Out of them 86 patients (57.3\%) were diagnosed with thrombocytopenia at or before 36 weeks of gestation, while 64 patients (42.7\%) were diagnosed after 36 weeks. Thirty patients (20\%) delivered at or before 36 weeks of gestation, whereas 120 patients (80\%) delivered after 36 weeks of gestation.

At the time of delivery, 44 patients (29.3\%) had mild thrombocytopenia, 99 patients (66\%) had moderate thrombocytopenia, and 7 patients (4.7\%) had severe thrombocytopenia.

Figure 1 shows comparative platelet counts at the time of diagnosis and delivery.

Preeclampsia was found to be the most common cause of thrombocytopenia in our study group, with 50 patients (33.3\%) presenting with thrombocytopenia and another 31 patients $(20.7 \%)$ further progressed and presented with HELLP syndrome. Gestational thrombocytopenia, being the second most common cause in our study, was found in 42 patients (28\%). Infectious causes such as dengue, malaria, and leptospirosis were found in 19 patients (12.7\%). Other causes of thrombocytopenia found in our study group were immune thrombocytopenic purpura (ITP) in 5 cases (3.3\%), heparininduced thrombocytopenia (HIT) in 2 cases (1.3\%), and aplastic anemia in 1 case (0.7\%) (Table 2 and Fig. 2). According to the cause of thrombocytopenia, mean gestational age at the time of diagnosis and delivery, mean platelet count, and lowest platelet count have been mentioned in Table 3.

Eighty-two patients (54.7\%) had normal vaginal deliveries, whereas 68 patients (45.3\%) underwent LSCS, of which 27 patients (18\%) were given GA and 41 patients (27.3\%) were given SA (Table 4). Patients given SA were of mild to moderate thrombocytopenia without any added complications. None of the patients given SA developed neurological or any other complications after the procedure till discharge. Patients with severe thrombocytopenia or with any complication especially hemorrhagic were administered GA.

Complications noted were APH in 11 patients (7.3\%), PPH in 24 patients (16\%), and 12 patients (8\%) required ICU admission amongst which there were 3 mortalities (2\%). Twenty-eight patients (18.7\%) required blood transfusion and 7 patients (4.7\%) required platelet transfusion (Table 5).

All the mentioned complications were observed in preeclampsia, preeclampsia with HELLP syndrome (82\%), and infectious causes mainly dengue fever (18\%). No complications were observed in gestational thrombocytopenia and no transfusion was required in these patients. Mortality was seen in 2 patients of preeclampsia with severe HELLP syndrome and 1 patient of dengue fever with coexisting malaria.

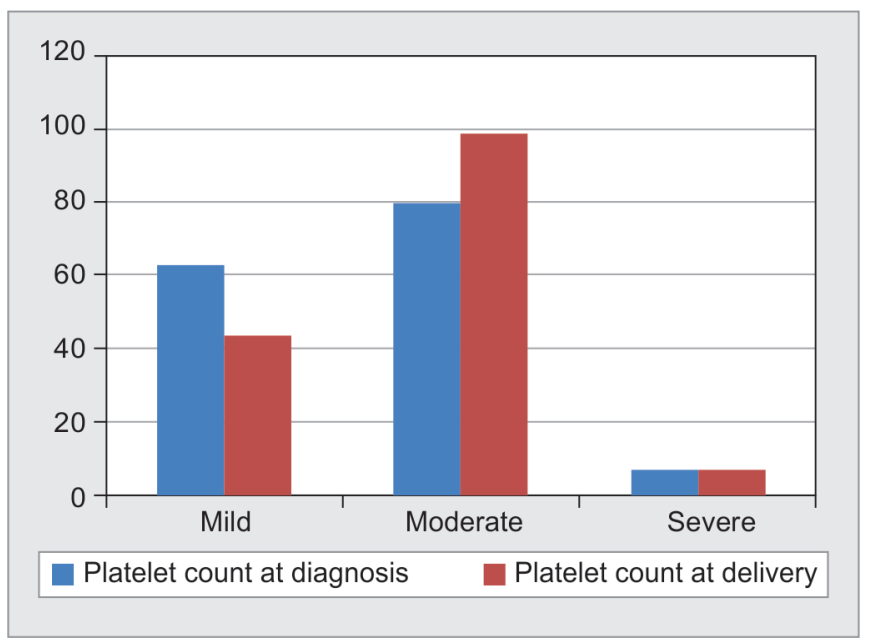

Fig. 1: Comparison and distribution of study group as per platelet count at time of diagnosis and time of delivery

Table 1: Age, gestational age, and platelet count at diagnosis and at time of delivery

\begin{tabular}{|c|c|c|c|c|c|c|c|}
\hline & $N$ & Mean & $\begin{array}{l}\text { Standard } \\
\text { deviation }\end{array}$ & Median & $\begin{array}{l}\text { Interquartile } \\
\text { range }\end{array}$ & Minimum & Maximum \\
\hline Age & 150 & 25.27 & 4.073 & 24.50 & 6.000 & 19 & 38 \\
\hline Gestational age at diagnosis & 150 & 35.66 & 2.807 & 36.00 & 4.000 & 30 & 40 \\
\hline Gestational age at delivery & 150 & 37.96 & 1.753 & 38.00 & 2.000 & 32 & 41 \\
\hline Platelet count at diagnosis & 150 & $97,712.67$ & $25,426.193$ & $96,000.00$ & $39,000.000$ & 6,000 & $1,44,000$ \\
\hline Platelet count at delivery & 150 & $95,309.33$ & $25,028.463$ & $92,000.00$ & $28,500.000$ & 7,000 & $1,43,000$ \\
\hline
\end{tabular}


Fifteen neonates (10\%) required NICU admission, while there was 1 intrauterine fetal death (IUFD) (0.7\%). Transfusion was required in 5 neonates (3.3\%).

\section{Discussion}

During the study period of 1 year, incidence of thrombocytopenia among the patients admitted to labor ward was $8 \%$ (150 patients). Nisha et al. mention that overall incidence of thrombocytopenia as $8.8 \%{ }^{2}$ Mean age of patients in our study was 25.27 years. In the study by Wang et al., the average age of the thrombocytopenic pregnant

Table 2: Distribution of study group as per cause of thrombocytopenia

\begin{tabular}{lrc}
\hline Cause of thrombocytopenia & Frequency & Percentage \\
\hline Aplastic anemia & 1 & 0.7 \\
Gestational & 42 & 28.0 \\
HIT & 2 & 1.3 \\
Infectious & 19 & 12.7 \\
ITP & 5 & 3.3 \\
Preeclampsia with hemolysis, elevated liver & 31 & 20.7 \\
enzyme, and low platelet count syndrome & & \\
Preeclampsia without hemolysis, elevated & 50 & 33.3 \\
liver enzyme, and low platelet count & & \\
Total & 150 & 100.0 \\
\hline
\end{tabular}

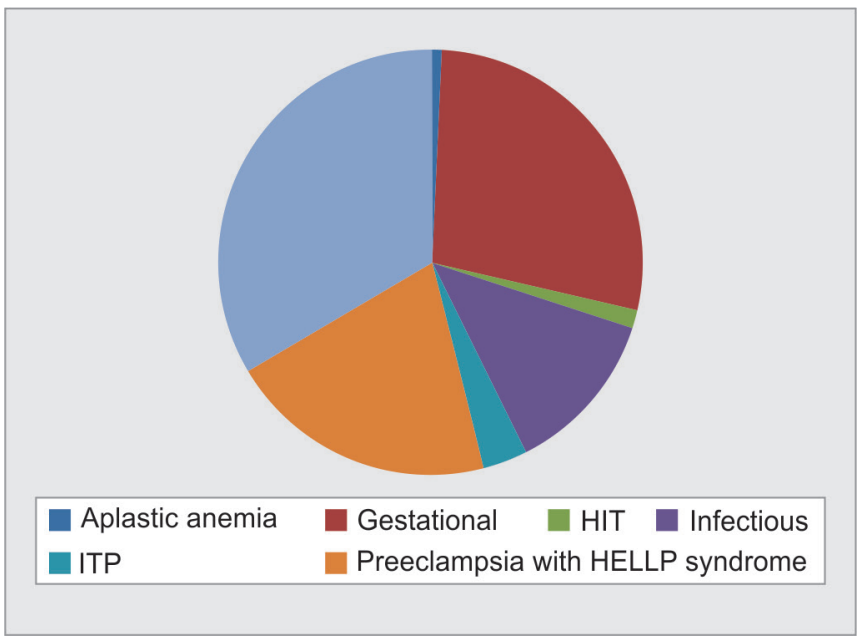

Fig. 2: Causes of thrombocytopenia patients was 29 years though Parnas et al. mention that older patients were at high risk for development of thrombocytopenia..$^{8,9}$

The present study was done only in patients admitted to labor ward and did not include early pregnancy. Average gestational age at diagnosis was 35.66 weeks and at delivery was 37.96 weeks. Mean platelet count at diagnosis was 97,712 and at delivery was 95,309. At the time of delivery, 44 patients (29.3\%) had mild thrombocytopenia, 99 patients (66\%) had moderate thrombocytopenia, and 7 patients (4.7\%) had severe thrombocytopenia.

Preeclampsia was found to be the most common cause of thrombocytopenia in our study group, with 50 patients $(33.3 \%)$ presenting with thrombocytopenia and another 31 patients (20.7\%) further progressed and presented with HELLP syndrome. Gestational thrombocytopenia, being the second most common cause in our study, was found in 42 patients (28\%). Infectious causes such as malaria, dengue, and leptospirosis were found in 19 patients (12.7\%). Other causes of thrombocytopenia found in our study group were ITP in 5 patients (3.3\%), HIT in 2 patients (1.3\%), and aplastic anemia in 1 patient $(0.7 \%)$.

Most common cause of thrombocytopenia was gestational in majority of the studies by Saino et al. (81\%), Parnas et al. (59.3\%), Chen et al. (63.5\%), and Brychtová et al. (79.55\%). ${ }^{3,9-11}$ Wang and Szkodny et al. (54\% and $87.5 \%$, respectively) found that main etiology of thrombocytopenia in the pregnant patients was ITP. ${ }^{8,12}$ Preeclampsia was found to be one of the common causes in all the studies which is concurrent with our findings. In the present study, we noted infectious diseases mainly vector borne diseases such as malaria, dengue, and leptospirosis (12.7\%) as one of the important causes. Kam et al. mention that malaria in pregnancy in their review article. ${ }^{5}$ In a study by Mbanya et al. found that thrombocytopenic pregnant patients had history of preeclampsia in $23.3 \%$ and malaria in $22.3 \% .^{13}$ In a study by Nisha et al., malaria-related thrombocytopenia was seen in $2.11 \%$ patients. $^{2}$ Also some Asian studies mention that vector borne diseases are common causes of thrombocytopenia in pregnancy. ${ }^{14-16}$ Thus, endemic diseases have been found to be a major risk factor for thrombocytopenia in pregnant patients in Indian subcontinent. Gernsheimer has summarized all the causes of thrombocytopenia and mentions viral infection as $<1 \%$ of all etiologies. ${ }^{1}$ There is significant contribution of infective and vector disease causing thrombocytopenia which was apparently absent in the studies from western countries. ${ }^{17}$ Thus, it appears that with different study populations even in Indian scenario, the causes could be different and may be due to regional influence; hence, a multi-centric study with larger sample size is necessary.

Table 3: Mean gestational age, mean platelet count, and lowest platelet count according to cause of thrombocytopenia

\begin{tabular}{lllllc}
\hline Cause of thrombocytopenia & $\begin{array}{l}\text { Mean gestational } \\
\text { age at diagnosis }\end{array}$ & $\begin{array}{l}\text { Mean gestational } \\
\text { age at delivery }\end{array}$ & $\begin{array}{l}\text { Mean platelet count } \\
\text { at diagnosis }\end{array}$ & $\begin{array}{l}\text { Mean platelet count } \\
\text { at delivery }\end{array}$ & $\begin{array}{l}\text { Lowest platelet count } \\
\text { at delivery }\end{array}$ \\
\hline Aplastic anemia & 32 & 32 & 6,000 & 7,000 & 7,000 \\
Gestational & 33.35 & 38.45 & $1,15,038.1$ & $1,11,964.3$ & 72,000 \\
HIT & 35 & 39.5 & $1,11,500$ & $1,02,000$ & 96,000 \\
Infectious & 38.89 & 38.89 & $84,578.94$ & $84,578.94$ & 40,000 \\
ITP & 37 & 39 & $1,02,000$ & $1,01,200$ & 94,000 \\
$\begin{array}{l}\text { Preeclampsia with hemolysis, } \\
\text { elevated liver enzyme, and low } \\
\text { platelet count syndrome }\end{array}$ & 36.09 & 37.51 & $86,516.12$ & $81,387.09$ & 46,000 \\
$\begin{array}{l}\text { Preeclampsia without hemolysis, } \\
\text { elevated liver enzyme, and low } \\
\text { platelet count }\end{array}$ & 36.06 & & & & \\
\hline
\end{tabular}


Table 4: Distribution of study group as per mode of delivery and type of anesthesia

\begin{tabular}{lcc}
\hline Mode of delivery & Frequency & Percentage \\
\hline Normal delivery & 82 & 54.7 \\
Cesarean section_general anesthesia & 27 & 18.0 \\
Cesarean section_-spinal anesthesia & 41 & 27.3 \\
Total & 150 & 100.0 \\
\hline
\end{tabular}

Table 5: Outcome, complications in study population

\begin{tabular}{llc}
\hline Outcome/complications & Frequency & Percentage \\
\hline ICU admission & 12 & 8 \\
Antepartum hemorrhage & 11 & 7.3 \\
Postpartum hemorrhage & 24 & 16.0 \\
Transfusion in mother & 28 & 18.7 \\
NICU admission & 15 & 10.0 \\
Transfusion in neonate & 5 & 3.3 \\
IUFD & 1 & 0.7 \\
Maternal mortality & 3 & 2 \\
\hline
\end{tabular}

Our study is the stepping-stone to prove the association of endemic diseases with increased incidence of thrombocytopenia in pregnant Indian patients. The current study widened the spectrum of the causes for thrombocytopenia in pregnant patients. Being tropical developing country, these conditions should be kept in mind while treating thrombocytopenia in pregnancy.

In the present study, mild to moderate thrombocytopenia was seen mainly in gestational thrombocytopenia with a mean platelet count of 1,11,964.3. Moderate to severe thrombocytopenia was observed mainly in preeclampsia, preeclampsia with HELLP syndrome (mean platelet count 81,387.09), and infectious etiology (mean platelet count 84,578.94). One case of aplastic anemia had lowest platelet count of 6,000. Studies have mentioned concurrent results stating gestational thrombocytopenia usually presents as mild thrombocytopenia without any adverse effect on either the mother or the fetus. ${ }^{2,6,3-18}$ Moderate to severe thrombocytopenia patients should be evaluated for other causes of thrombocytopenia, and watchful expectancy for adverse events is needed.

In the present study, 30 patients (20\%) delivered at or before 36 weeks of gestation. Nisha et al. mention that preterm delivery in thrombocytopenia patients due to the associated obstetric and medical complications. ${ }^{2}$ In the study by Chen et al., preterm deliveries occurred in $18.2 \%$ patients. ${ }^{10}$

In the present study, 82 patients (54.7\%) had normal vaginal deliveries, whereas 68 patients (45.3\%) underwent LSCS. Lower segment cesarean section was indicated only for obstetric indications. Majority of the studies mentioned concurrent results. ${ }^{2,6}$ In the study by Nisha et al., normal vaginal deliveries were in $61.54 \%$ and cesarean section was done in $36.26 \%$ patients. $^{2}$

Contrasting results were found in the study by Wang et al., where cesarean section was performed in $85.18 \%$ patients. ${ }^{8}$ Gernsheimer also affirmed that mode of delivery in thrombocytopenia is based on obstetric indications, with avoidance of procedures like forceps which may increase hemorrhagic risk to the fetus. ${ }^{1}$ Varghese et al. mention that higher prevalence of preterm deliveries, labor induction, and cesarean section in patients of moderate to severe thrombocytopenia. ${ }^{17}$
The choice of anesthetic technique for LSCS largely depends on the associated obstetric complications, other significant medical history, and coagulation status. Regional (spinal or epidural) anesthesia is considered standard of care for parturient unless contraindicated. Severe thrombocytopenia is considered contraindication for neuraxial anesthesia due to potential risk of development of an epidural or subdural hematoma., ${ }^{5,21}$ It although is very rare complication with incidence ranging from $0 \%$ to $0.6 \%$ but dreaded one that may result in permanent neurologic injury. ${ }^{19}$ In the current study, 68 required LSCS, among them 27 patients (18\%) were given GA and 41 patients (27.3\%) were given SA. SA was administered to patients of mild to moderate thrombocytopenia without any added complications and none of them developed neurological or any other complications after the procedure till discharge. Patients with severe thrombocytopenia or with any complication especially hemorrhagic were administered GA.

In patients with thrombocytopenia not only platelet count but also their function determines the safety of regional anesthesia. Platelet count of $1,00,000 / \mu \mathrm{L}$ is recommended as "safe" for performing an epidural blockade and $80,000 / \mu \mathrm{L}$ for $\mathrm{SA} .{ }^{5,6,19,20}$ Some hematologists suggest that a platelet count of $>50,000 / \mu \mathrm{L}$ is safe for surgery and neuraxial blockade, provided platelet function is normal. ${ }^{5,22}$ However, Khellaf mentions that a platelet count of $50,000 / \mu \mathrm{L}$ is required for the delivery and of $75,000 / \mu \mathrm{L}$ in case of SA. ${ }^{6}$

In a multicenter retrospective cohort study by Goodier emphasizes the relative maternal safety of neuraxial anesthesia in parturients with mild thrombocytopenia. ${ }^{19}$

Lee et al. confirmed that there is considerable increase in the number of thrombocytopenic parturients who received neuraxial techniques without complication and platelet count more than $70,000 / \mu \mathrm{L}$ is considered safe. ${ }^{21}$ Even though there is no consensus on the acceptable platelet count required to safely perform neuraxial anesthesia, recent literature recommends that lower thresholds may be safe in pregnant women. Further studies are needed for standardization of anesthesia practice to safely perform neuraxial anesthesia in thrombocytopenic parturients. ${ }^{19-22}$

At our institute, standard practice is to give SA with platelet counts $>80,000 / \mu \mathrm{L}$ and epidural with platelet counts $>1,00,000 / \mu \mathrm{L}$. Gernsheimer also mentions similar protocol in thrombocytopenia. ${ }^{1}$

In the current study, 11 patients (7.3\%) developed APH, while 24 (16\%) developed $\mathrm{PPH}, 28$ patients (18.7\%) required blood transfusion, and 7 (4.7\%) required platelet transfusion. While 12 patients (8\%) required ICU admission amongst which there were 3 mortalities (2\%).

Mortality was seen in 2 patients of preeclampsia with severe HELLP syndrome and 1 patient of vector borne disease who had coexistent dengue hemorrhagic fever with malaria. Fifteen neonates (10\%) required NICU admission, while there was 1 IUFD (0.7\%). Transfusion was required in 5 neonates (3.3\%).

All the mentioned complications in mother and neonates were observed in preeclampsia, preeclampsia with HELLP syndrome (82\%), and infectious causes mainly malaria and dengue fever (18\%). No complications were observed in gestational thrombocytopenia and no transfusion was required in these patients. Also complications were only observed in moderate to severe thrombocytopenia.

Varghese et al. mention that higher incidence of complications like preterm deliveries, requirement of blood transfusion, and intrauterine growth restriction (IUGR) in presence of moderate to severe thrombocytopenia. ${ }^{18} \mathrm{Harde}$ et al. mention that hemorrhage 
and hypertensive disorder of pregnancy were leading indications for ICU admission.

Our results were concurrent with other authors who mentioned outcome was good in cases of gestational thrombocytopenia and adverse pregnancy outcome was associated with other etiologies such as preeclampsia and HELLP syndrome. ${ }^{9,16-18,22}$

Nisha et al. observed higher incidence of PPH (9.89\%) in medical thrombocytopenia and morbidity and mortality only in medical and obstetric thrombocytopenia. ${ }^{2}$ Dikman et al. confirm that thrombocytopenic women may have adverse maternal and neonatal outcomes with $\mathrm{PPH}$, prematurity, and limited regional anesthesia. ${ }^{20}$

Chitra has reported dengue fever in pregnancy may be associated with very severe thrombocytopenia and an increased incidence of pre-term deliveries, low birth weight, preeclampsia, and cesarean sections. ${ }^{14}$

Thus, moderate to severe thrombocytopenia patients especially due to preeclampsia, HELLP syndrome, and infectious causes should be kept under cautious observation, and prevention and preparedness for managing complications with expert multispecialty involvement should be emphasized.

The study had its share of limitations as it was observational single center study limited to patients nearing term and did not include early pregnancy.

From the experience of the present study on thrombocytopenia in pregnant Indian females in third trimester mainly during labor and delivery we conclude as follows: preeclampsia (with or without HELLP syndrome) was found to be the most common cause of thrombocytopenia followed by gestational thrombocytopenia and infectious causes. Other causes noted were ITP, HIT, and aplastic anemia. Contribution of vector borne infections such as malaria, dengue, and leptospirosis was found to be one of the very important emerging causes in India. Therefore, the association of endemic diseases with increased incidence of thrombocytopenia in pregnant Indian patients should be kept in mind. The current study widened the spectrum of the causes for thrombocytopenia in pregnant patients. Gestational thrombocytopenia was associated with mild to moderate thrombocytopenia and was without any complications. Moderate to severe thrombocytopenia was observed mainly in preeclampsia, preeclampsia with HELLP syndrome, and infectious etiology and was associated with complications like APH, PPH, ICU admissions, mortality, and fetal complications. Mode of delivery in thrombocytopenia is based on obstetric indications only, and maternal safety of neuraxial anesthesia in parturients with mild thrombocytopenia is emphasized. Moderate to severe thrombocytopenia patients should be evaluated for other causes of thrombocytopenia, and vigilant observation and preparedness for adverse events is considered indispensable.

Thrombocytopenia in pregnancy is a challenging task to manage on all fronts. It starts with correct diagnosis from variety of causes. It is crucial to distinguish benign gestational from preeclampsia HELLP and infective causes which have the potential for serious morbidity and mortality. As a step toward reducing maternal morbidity and mortality, we recommend while treating thrombocytopenia in pregnancy in tropical developing countries, vector borne diseases should be considered as an important differential diagnosis and early vigilance in cases of febrile illness is must. Education and awareness among expectant mothers regarding seeking early medical attention in cases of febrile illness should go a long way in reducing maternal morbidity and mortality. Prevention and preparedness for managing anticipated complications should be emphasized. Expert teamwork involving obstetricians, hematologists, and anesthesiologist is fundamental for optimal management of the mother and fetus.

\section{References}

1. Gernsheimer T, James AH, Stasi R. How I treat thrombocytopenia in pregnancy. Blood 2013;121(1):38-47. DOI: 10.1182/blood-2012-08448944.

2. Nisha S, Dhakad A, Uma S, Tripathi AK, Pushpalata S. Prevalence and characterization of thrombocytopenia in pregnancy in Indian women. Indian J Hematol Blood Transfus 2012;28(2):77-81. DOI: 10.1007/s12288-011-0107-x.

3. SainoS,KekomakiR, Riikonon S, TeramoK. Maternal thrombocytopenia at term: a population-based study. Acta Obstet Gynecol Scand 2000;79(9):744-749. DOI: 10.1034/j.1600-0412.2000.079009744.x.

4. Won YW, Moon W, Yun YS, Oh HS, Choi JH, Lee YY, et al. Clinical aspects of pregnancy and delivery in patients with chronic idiopathic thrombocytopenic purpura (ITP). Korean J Intern Med 2005;20(2):129. DOI: $10.3904 / \mathrm{kjim} .2005 .20 .2 .129$.

5. Kam PC, Thompson SA, Liew AC. Review article, thrombocytopenia in the parturient. Anaesthesia 2004;59(3):255-264. DOI: 10.1111/j.13652044.2004.03576.x.

6. KhellafM, Loustau V,Bierling P,Michel M, Godeau B. Thrombocytopenia and pregnancy. Rev Med Interne 2012;33(8):446-452. DOI: 10.1016/ j.revmed.2012.05.011.

7. Harde M, Dave S, Vasave RR, Gujjar P, Bhadade R. Lower segment cesarean section in a patient with severe thrombocytopenia and pregnancy-induced hypertension. J Anaesthesiol Clin Pharmacol 2013;29(3):387-389. DOI: 10.4103/0970-9185.117110.

8. Wang DP, Liang MY, Wang SM. Clinical analysis of pregnancy complicated with severe thrombocytopenia. Zhonghua Fu Chan KeZaZhi 2010;45(6):401-405.

9. Parnas M, Sheiner E, Shoham-Vardi I, Burstein E, Yermiahu T, Levi I, et al. Moderate to severe thrombocytopenia during pregnancy. Eur J Obstet Gynecol Reprod Biol 2006;128(1-2):163-168. DOI: 10.1016/j. ejogrb.2005.12.031.

10. Chen Z, Liang MY, Wang JL. Etiology and clinical characteristics of pregnancy-emerged thrombocytopenia. Zhonghua Fu Chan KeZaZhi 2011;46(11):834-839.

11. Brychtová $P$, Procházka $M$, Lattová V, Lubušký $M$, Procházková J, Slavík L, et al. Occurrence, etiology and clinical significance of thrombocytopenia in pregnancy. Ceska Gynekol 2013;78(6):560-565.

12. Szkodny E, Sikora J, Bakon I, Zietek J. Pregnancy and delivery in women with thrombocytopenia. Ginekol Pol 2001;72(12A):1296-1299.

13. Mbanya D, TayouTagny C, Takoeta E, Mbu R, Kaptue L. Factors associated with thrombocytopenia among pregnant women in Cameroon. Sante 2007;17(4):213-217. DOI: 10.1684/san.2007.0085.

14. Chitra TV, Panicker S. Maternal and fetal outcome of dengue fever in pregnancy. J Vector Borne Dis 2011;48(4):210-213.

15. Ismail NA, Kampan N, Mahdy Z, Abdul Jamil MA, Mohd Razi ZR Dengue in pregnancy. Southeast Asian J Trop Med Public Health 2006;37(4):681-683.

16. Jeffrey $A L$, Lance DM. Thrombocytopenia in pregnancy. J Am Board Fam Pract 2002;15:290-297.

17. Varghese S, Kour G, Dhar T. Thrombocytopenia in pregnancy in a tertiary care hospital: a retrospective study. Int J Reprod Contracept Obstet Gynecol 2016;5:1532-1535. DOI: 10.18203/2320-1770. ijrcog20161318.

18. Kasai J, Aoki S, Kamiya N, Hasegawa Y, Kurasawa K, Takahashi T, et al. Clinical features of gestational thrombocytopenia difficult to differentiate from immune thrombocytopenia diagnosed during pregnancy. J Obstet Gynaecol Res 2015;41(1):44-49. DOI: 10.1111/ jog.12496.

19. Goodier CG, Lu JT, Hebbar L, Segal BS, GoetzI L. Neuraxial anesthesia in parturients with thrombocytopenia: a multisite retrospective 
cohort study. Anesth Analg 2015;121(4):988-991. DOI: 10.1213/ ANE.0000000000000882.

20. Dikman D, Elstein D, Levi GS, Granovsky-Grisaru S, Samueloff A, Gozal $Y$, et al. Effect of thrombocytopenia on mode of analgesia/anesthesia and maternal and neonatal outcomes. J Matern Fetal Neonatal Med 2014;27(6):597-602. DOI: 10.3109/14767058.2013.836483.

21. Lee LO, Bateman BT, Kheterpal S, Klumpner TT, Housey M, Aziz MF. Risk of epidural hematoma after neuraxial techniques in thrombocytopenic parturients. Anesthesiology 2017;126(6): 1053-1064. DOI: 10.1097/ALN.0000000000001630.

22. Ozdemir S, Gorkemli H, Acar A, Celik C, Kayikcioglu E. A comparison of maternal and fetal outcomes of pregnancies complicated by moderate to severe thrombocytopenia caused by gestational thrombocytopenia preeclampsia/HELLP syndrome and immune thrombocytopenic purpura. Gynecology Obstetrics \& Reproductive Medicine 2016;14(3):154-158. 